Applied Mathematical Sciences, Vol. 2, 2008, no. 47, 2323 - 2339

\title{
Numerical Simulation of a Diffusion Type Evolutionary Stock Market Model
}

\author{
Walailuck Chavanasporn \\ School of Economics and Finance, University of St.Andrews \\ St Salvator's College, St Andrews, Fife KY16 9AL, Scotland, UK \\ wc55@st-andrews.ac.uk \\ Christian-Oliver Ewald \\ School of Economics and Finance, University of St.Andrews \\ St Salvator's College, St Andrews, Fife KY16 9AL, Scotland, UK \\ c.o.ewald@st-andrews.ac.uk
}

\begin{abstract}
We adapt the evolutionary stock market model from Evstigneev, Hens, Schenk-Hoppé (2006) to a continuous time framework, where uncertainty in dividends is produced by a single Wiener process. The setup is therefore significantly different from Yang and Ewald (2008), who also study continuous time, but remain within the framework of random dynamical systems of non-diffusive type. For the case of fix-mix strategies we derive the stochastic differential equation which determines the evolution of the wealth processes of the various market players. These stochastic differential equations are highly non-linear and we find that it is impossible to solve them analytically. Instead we simulate the wealth dynamic for various initial setups of the market. A detailed discussion of our observations from the simulations is given.
\end{abstract}

Mathematics Subject Classification: 91B28 (47H40 60H25)

Keywords: Behavioral Finance, Evolutionary Finance, Wealth dynamics

\section{Introduction}

According to the expected discounted dividends model; one of the fundamental models used widely in finance, the rational and fair value of common stocks is given by the discounted sum of future dividends paid out by the company. It is found that in the long run the trend of stock prices coincides with the 
trend of the dividends paid by the companies while the prices can considerably deviate from their dividend fundamentals over shorter horizons.

Two stock market models are presented in this paper. The first one is the discrete time evolutionary stock market model set up by Evstigneev, Hens and Schenk-Hoppé (2006). The second model is an adaptation of the first one in a continuous time framework where randomness produced by a Wiener process. Evstigneev, Hens and Schenk-Hoppé study an incomplete asset market with heterogeneous population of portfolio rules in discrete time. The finite number of investment rules manage capital by repeated reinvesting in a fixed set of longlived assets. Dividend payments in each period are according to the realization of a stationary Markov process. Moreover, the exogenous wealth increases due to the dividends, trading strategies face endogenously determined capital gains or losses.

The trading strategies compete for market capital that is given by the total value of all assets in each period in time. Using random dynamical systems theory, they derive necessary and sufficient conditions for the evolutionary stability of portfolio rules. In case of Markov payoffs, Evstigneev, Hens and Schenk-Hoppé discover that the local stability conditions lead to a simple portfolio rule that is the unique evolutionary stable strategy.

In a continuous time framework, we set up a model by supposing that the company pays dividends continuously and the processes in our model are all of Itô-type. Due to the complexity of the model, the problem cannot be solved analytically. Even for the derivation of a tractable wealth dynamics, severe restriction on the type of admissible strategies have to be made. We indicate the problem that arises due to the quadratic variation term stemming from the strategy itself, which then in principle would lead to a singular optimal control problem. We don't continue along this line, but instead restrict our investigation to fix-mix strategies. This strategies have also been considered in Evstigneev, Hens and Schenk-Hoppé (2006) as well as Yang and Ewald (2008) and include important cases such as the Merton rule for example. The results of various numerical simulation and experiments are presented and discussed. The simulations contain 3 different cases relating to different initial wealth distributions. The results vary depending upon the initial market shares of each trader and the strategies they apply. Our simulations show that in some cases, one agent can find a dominant strategy that cannot be invaded from any strategy another agent uses. There are also cases where one investor gains total market share while the other goes bankrupt. However, there are cases that the market shares are allocated to each investor equally.

The remainder is organized as follows. The next section illustrates the evolutionary stock market model in discrete time which is set up by Evstigneev, Hens and Schenk-Hoppé (2006). The model comprises an infinite time horizon asset market model with long-lived assets and a single perishable consumption 
good. In this model, heterogeneous portfolio rules adapted to the information filtration are introduced. In section 3, we modify the model in a way that dividends are paid continuously and all processes are of Itô-type. The results of a numerical simulation are provided and documented in section 4. Details of the derivation of the wealth dynamics have been put to the appendix.

\section{An Evolutionary Stock Market Model in Dis- crete Time}

In this section, we present a brief review of the evolutionary stock market model developed by Evstigneev, Hens and Schenk-Hoppé (2006). These authors consider an asset market model in discrete time with $K \geq 1$ long-lived assets and a single perishable consumption good. A dividend per share of each asset $k=1, \ldots, K$ is paid at the beginning of every period and before trade takes place in the period. The total dividend paid to all shareholders of asset $k$ at the beginning of period $t$ is denoted by $D_{t}^{k} \geq 0$. $\omega^{t}=\left(\ldots, \omega_{0}, \ldots, \omega_{t}\right)$ where $\omega_{t} \in S$ denotes the states revealed at the beginning of period $t$. There are $I \geq 2$ investors who use portfolio rules denoted by $\lambda_{t}^{i}\left(\omega^{t}\right)=\left(\lambda_{t, k}^{i}\left(\omega^{t}\right)\right)_{k=0, \ldots K}$ with $0 \leq \lambda_{t, k}^{i}\left(\omega^{t}\right) \leq 1$ for all $k$ and $\sum_{k=0}^{K} \lambda_{t, k}^{i}\left(\omega^{t}\right)=1$. The interpretation is that $\lambda_{t, k}^{i}\left(\omega^{t}\right)$ is the fraction of the wealth investor $i$ assigns to the purchase of the asset $k$ in period $t$. Investment strategies are assumed to be distinct across investors.

The units of assets $(k=1, \ldots, K)$ or units of cash $(k=0)$ that investor $i$ holds can be computed from

$$
\theta_{t, k}^{i}=\frac{\lambda_{t, k}^{i}\left(\omega^{t}\right) w_{t}^{i}}{p_{t}^{k}} ; k=0,1, \ldots, K
$$

given portfolio rules $\lambda_{t, k}^{i}\left(\omega^{t}\right)$ and wealth $w_{t}^{i}$. The price $p_{t}^{k}$ is the market clearing price of asset $k$ in period $t$. The initial supply of every asset $k, s_{0}^{k}$, is normalized to 1 , and the supply remains constant at any period in time, i.e. $s_{t}^{k}=s_{0}^{k}$. In addition, the supply of cash $s_{t}^{0}$ is given by the total dividends of all assets. Hence, $\theta_{t, k}^{i}$ can be interpreted as the number of all shares issued of asset $k$ that investor $i$ purchases. The price for cash is normalized to one, i.e. $p_{t}^{0}=1$ in every period $t$, and the same as the price of the consumption good. It is also concluded that the market equilibrium conditions for cash and long-lived assets for any portfolio holding of agents are $\sum_{i=1}^{I} \theta_{t, k}^{i}=s_{t}^{k} ; k=0,1, \ldots, K$.

Since $\sum_{k=0}^{K} \lambda_{t, k}^{i}\left(\omega^{t}\right)=1$, the budget constraint for investor $i$ in every period 
$t=0,1, \ldots$ is

$$
\sum_{k=0}^{K} p_{t}^{k} \theta_{t, k}^{i}=w_{t}^{i}
$$

Due to the fact that the consumption good is perishable; the dynamics of wealth is affected by dividend payments and capital gains. So the wealth of investor $i$ at the beginning of period $t+1$ and after dividends are paid (before the trade takes place) is

$$
w_{t+1}^{i}=\sum_{k=1}^{K}\left(D_{t+1}^{k}\left(\omega^{t+1}\right)+p_{t+1}^{k}\right) \theta_{t, k}^{i}
$$

From $\theta_{t, k}^{i}=\frac{\lambda_{t, k}^{i}\left(\omega^{t}\right) w_{t}^{i}}{p_{t}^{k}}$, and $\sum_{i=1}^{I} \theta_{t, k}^{i}=1$, the market clearing price for the risky assets $(k \geq 1)$ can be derived as

$$
p_{t}^{k}=\sum_{i=1}^{I} \lambda_{t, k}^{i}\left(\omega^{t}\right) w_{t}^{i}
$$

\section{An Evolutionary Stock Market Model in Con- tinuous Time}

In this section, we modify the evolutionary stock market model described in the previous section assuming that dividends are paid continuously and then set up a stock market in continuous time model. Let $D_{t}$ denote the dividend paid in the period $[t-\Delta t, t]$.

In order to adapt the model into continuous time we consider the wealth process and price process when time increments are going to zero. The wealth of investor $i$ at the beginning of period $t+\Delta t$ and after dividends are paid is

$$
w_{t+\Delta t}^{i}=\sum_{k=1}^{K}\left(D_{t+\Delta t}^{k}\left(\omega^{t+\Delta t}\right)+p_{t+\Delta t}^{k}\right) \theta_{t, k}^{i} .
$$

This gives

$$
\begin{aligned}
w_{t+\Delta t}^{i}-w_{t}^{i}= & \sum_{k=1}^{K}\left[D_{t+\Delta t}^{k}\left(\omega^{t+\Delta t}\right) \theta_{t, k}^{i}+p_{t+\Delta t}^{k} \theta_{t, k}^{i}-D_{t}^{k}\left(\omega^{t}\right) \theta_{t-\Delta t, k}^{i}-p_{t}^{k} \theta_{t-\Delta t, k}^{i}\right] \\
= & \sum_{k=1}^{K}\left[\left(D_{t+\Delta t}^{k}\left(\omega^{t+\Delta t}\right)-D_{t}^{k}\left(\omega^{t}\right)\right) \theta_{t, k}^{i}+\left(p_{t+\Delta t}^{k}-p_{t}^{k}\right) \theta_{t, k}^{i}\right. \\
& \left.+\left(D_{t}^{k}\left(\omega^{t}\right)+p_{t}^{k}\right) \Delta \theta_{t, k}^{i}\right] .
\end{aligned}
$$


Letting $\Delta t$ go to zero we obtain the following continuous time interpretation of the wealth dynamic:

$$
d w_{t}^{i}=\sum_{k=1}^{K}\left[\left(d D_{t}^{k}\left(\omega^{t}\right)+d p_{t}^{k}\right) \theta_{t, k}^{i}+\left(D_{t}^{k}\left(\omega^{t}\right)+p_{t}^{k}\right) d \theta_{t, k}^{i}\right]
$$

or alternatively in integrated form

$$
w_{t}^{i}=\int_{0}^{t} \sum_{k=1}^{K}\left[\left(d D_{s}^{k}\left(\omega^{s}\right)+d p_{s}^{k}\right) \theta_{s, k}^{i}+\left(D_{s}^{k}\left(\omega^{s}\right)+p_{s}^{k}\right) d \theta_{s, k}^{i}\right] .
$$

As the $\theta_{s, k}^{i}$ are the choice variables, without further restriction we would formally obtain some sort of singular stochastic optimal control problem. These problems are typically very hard to deal with and we will make suitable restrictions to avoid this. On the other side, the market clearing price at period $t+\Delta t$ is

$$
p_{t+\Delta t}^{k}=\sum_{i=1}^{I} \lambda_{t+\Delta t, k}^{i}\left(\omega^{t+\Delta t}\right) w_{t+\Delta t}^{i}
$$

and the increment is given by

$$
\begin{aligned}
p_{t+\Delta t}^{k}-p_{t}^{k}= & \sum_{i=1}^{I}\left[\lambda_{t+\Delta t, k}^{i}\left(\omega^{t+\Delta t}\right) w_{t+\Delta t}^{i}-\lambda_{t, k}^{i}\left(\omega^{t}\right) w_{t}^{i}\right] \\
= & \sum_{i=1}^{I}\left[\lambda_{t+\Delta t, k}^{i}\left(\omega^{t+\Delta t}\right) w_{t+\Delta t}^{i}-\lambda_{t, k}^{i}\left(\omega^{t}\right) w_{t}^{i}\right. \\
& \left.+w_{t+\Delta t}^{i} \lambda_{t, k}^{i}\left(\omega^{t}\right)-w_{t+\Delta t}^{i} \lambda_{t, k}^{i}\left(\omega^{t}\right)\right] \\
= & \sum_{i=1}^{I}\left[\lambda_{t, k}^{i}\left(\omega^{t}\right)\left(w_{t+\Delta t}^{i}-w_{t}^{i}\right)+w_{t+\Delta t}^{i}\left(\lambda_{t+\Delta t, k}^{i}\left(\omega^{t+\Delta t}\right)-\lambda_{t, k}^{i}\left(\omega^{t}\right)\right)\right] .
\end{aligned}
$$

Letting $\Delta t$ go to zero we obtain the following continuous time interpretation of the price dynamic:

$$
\begin{gathered}
d p_{t}^{k}=\sum_{i=1}^{I}\left[\lambda_{t, k}^{i}\left(\omega^{t}\right) d w_{t}^{i}+w_{t}^{i} d \lambda_{t, k}^{i}\left(\omega^{t}\right)\right] \\
p_{t}^{k}=\int_{0}^{t} \sum_{i=1}^{I}\left[\lambda_{s, k}^{i}\left(\omega^{s}\right) d w_{s}^{i}+w_{s}^{i} d \lambda_{s, k}^{i}\left(\omega^{s}\right)\right]
\end{gathered}
$$

Here the term $d \lambda_{s, k}^{i}\left(\omega^{s}\right)$ is worrying, as in a general framework it will produce unwanted quadratic variation which would potentially lead us out of the 
class of diffusion type models. For this reason we restrict our investigations to so called fix-mix strategies, which are constant in time and state. This restriction is admittingly restrictive, on the other side classical strategies such as the Merton-rule and various fundamentalist strategies do well classify as such. The consequence of this assumption is that $d \lambda_{t, k}^{i}\left(\omega^{t}\right)=0$. Furthermore we can write $\lambda_{k}^{i}=\lambda_{t, k}^{i}\left(\omega^{t}\right)$.

Applying the Itô formula, we get

$$
\begin{aligned}
d \theta_{t, k}^{i}= & \frac{\partial \theta_{t, k}^{i}}{\partial w_{t}^{i}} d w_{t}^{i}+\frac{1}{2} \frac{\partial^{2} \theta_{t, k}^{i}}{\left(\partial w_{t}^{i}\right)^{2}}\left(d w_{t}^{i}\right)^{2} \\
= & \frac{\left(\sum_{i=1}^{I} \lambda_{k}^{i} w_{t}^{i}\right)\left(\lambda_{k}^{i}\right)-\left(\lambda_{k}^{i} w_{t}^{i}\right)\left(\sum_{i=1}^{I} \lambda_{k}^{i}\right)}{\left(\sum_{i=1}^{I} \lambda_{k}^{i} w_{t}^{i}\right)^{2}} d w_{t}^{i} \\
& -\left[\frac{\left\{\left(\sum_{i=1}^{I} \lambda_{k}^{i} w_{t}^{i}\right)\left(\lambda_{k}^{i}\right)-\left(\lambda_{k}^{i} w_{t}^{i}\right)\left(\sum_{i=1}^{I} \lambda_{k}^{i}\right)\right\}\left(\sum_{i=1}^{I} \lambda_{k}^{i}\right)}{\left(\sum_{i=1}^{I} \lambda_{k}^{i} w_{t}^{i}\right)^{3}}\right]\left(d w_{t}^{i}\right)^{2}
\end{aligned}
$$

We also obtain

$$
d p_{t}^{k}=\sum_{i=1}^{I}\left[\lambda_{k}^{i}\left(\omega^{t}\right) d w_{t}^{i}\right]
$$

To simplify the following discussion we are now considering the special case of two investors and two assets, one riskless asset, called bond, and one risky asset, called stock. The assets pay continuous dividends. Hence, there are two dividend processes, two wealth processes and two price processes. We suppose that the processes are in the form of Itô-type.

The two price processes satisfy

$$
d p_{t}^{k}=\sum_{i=1}^{2}\left[\lambda_{k}^{i} d w_{t}^{i}\right], k=1,2,
$$

and therefore $p_{t}^{k}=\sum_{i=1}^{2}\left[\lambda_{k}^{i} w_{t}^{i}\right] ; k=1,2$.

The dividend processes of the bond and stock are given by

$$
d D_{t}^{1}=r d t
$$


and

$$
d D_{t}^{2}=\alpha D_{t}^{2} d t+\sigma D_{t}^{2} d B(t)
$$

where $B(t)$ denotes the standard Brownian Motion. In the following we derive the stochastic differential equations which characterize the evolution of the wealth of the two investors. We assume that they are of diffusion-type, which means they can be written in the following form

$$
d w_{t}^{1}=\beta_{1}^{1}\left(w_{t}, \lambda_{k}\right) d t+\beta_{2}^{1}\left(w_{t}, \lambda_{k}\right) d B(t)
$$

and

$$
d w_{t}^{2}=\beta_{1}^{2}\left(w_{t}, \lambda_{k}\right) d t+\beta_{2}^{2}\left(w_{t}, \lambda_{k}\right) d B(t)
$$

with $w_{t}=\left(w_{t}^{1}, w_{t}^{2}\right)$ and $\lambda_{k}=\left(\lambda_{k}^{1}, \lambda_{k}^{2}\right)$. We will identify the unknown functions $\beta_{1}^{1}, \beta_{2}^{1}, \beta_{1}^{2}$ and $\beta_{2}^{2}$ by comparison with (5), using (7) and (9). It is clear that the smaller the consumption rate, the higher the growth rate of wealth. Therefore, the following we assume that all investors have the same rate of consumption, i.e. $\lambda_{0}^{i}\left(\omega^{t}\right)=\lambda_{0}\left(\omega^{t}\right)$. This assumption is made in order to make the study unbiased in favor of investor with a high saving rate. In fact this assumption has also been made in Evstigneev, Hens and Schenk-Hoppé (2006) and Yang and Ewald (2008). To find $\beta_{1}^{1}, \beta_{2}^{1}, \beta_{1}^{2}$ and $\beta_{2}^{2}$, we apply the Itô formula and substitute all processes we have. $\beta_{1}^{1}, \beta_{2}^{1}, \beta_{1}^{2}$ and $\beta_{2}^{2}$ are found eventually as shown in the four following equations. The details of the computation of all $\beta$ 's are presented in the appendix.

$$
\begin{aligned}
& \beta_{2}^{1}= \frac{\sigma w_{t}^{1}\left(\frac{\lambda_{2}^{1}}{P_{2}}-\frac{C}{W A-\lambda_{0}}\right)}{1-w_{t}^{1} C\left(\frac{W A+\lambda_{0}}{W A-\lambda_{0}}\right)-w_{t}^{1}\left(\frac{\left(\lambda_{1}^{1}\right)^{2}}{P_{1}}+\frac{\left(\lambda_{2}^{1}\right)^{2}}{P_{2}}\right)+W A} \\
& \beta_{2}^{2}=\frac{W A+\lambda_{0}}{W A-\lambda_{0}} \beta_{2}^{1}-\frac{\sigma}{W A-\lambda_{0}} \\
& \beta_{1}^{1}= \frac{1}{1-w_{t}^{1} C\left(\frac{W A+\lambda_{0}}{W A-\lambda_{0}}\right)-w_{t}^{1}\left(\frac{\left(\lambda_{1}^{1}\right)^{2}}{P_{1}}+\frac{\left(\lambda_{2}^{1}\right)^{2}}{P_{2}}\right)+W A} \\
&+\frac{w_{t}^{1} C}{W A-\lambda_{0}}\left\{W\left(A^{1} E^{1}+A^{2} E^{2}\right)\left[\left(\beta_{2}^{2}\right)^{2}-\left(\beta_{2}^{1}\right)^{2}\right]-r-\alpha\right\} \\
& \beta_{1}^{2}=\left(\frac{W A+\lambda_{0}}{W A-\lambda_{0}}\right) \beta_{1}^{1}+\frac{W\left(A^{1} E^{1}+A^{2} E^{2}\right)}{W A-\lambda_{0}}\left[\left(\beta_{2}^{2}\right)^{2}-\left(\beta_{2}^{1}\right)^{2}\right] \\
&\left.\left.-\frac{\alpha}{W A-\lambda_{0}}-\frac{\alpha}{W A-\lambda_{0}^{1} w_{t}^{1}} P_{2}\right)+W\left(A^{1} E^{1}+A^{2} E^{2}\right)\left(\beta_{2}^{1}\right)^{2}\right]
\end{aligned}
$$


where $W=w_{t}^{1}-w_{t}^{2}, P_{k}=\lambda_{k}^{1} w_{t}^{1}+\lambda_{k}^{2} w_{t}^{2}, A^{k}=\lambda_{k}^{1} \lambda_{k}^{2} \frac{D_{t}^{k}+P_{k}}{\left(P_{k}\right)^{2}}$ and $A=A^{1}+A^{2}$, $C=\frac{\lambda_{1}^{1} \lambda_{1}^{2}}{P_{1}}+\frac{\lambda_{2}^{1} \lambda_{2}^{2}}{P_{2}}, E^{k}=\frac{\lambda_{k}^{1}+\lambda_{k}^{2}}{P_{k}}$.

It is obvious that the complexity of the equations forbids an analytical solution to the wealth dynamics.

\section{Numerical Simulations and Experiments}

The analytic solution of the two dividend processes $d D_{t}^{1}=r d t$ and $d D_{t}^{2}=$ $\alpha D_{t}^{2} d t+\sigma D_{t}^{2} d B(t)$ with the initial conditions $D_{0}^{1}=0$ and $D_{0}^{2}=1$ are

$$
D_{t}^{1}=r t
$$

and

$$
D_{t}^{2}=\exp \left(\left(\alpha-\frac{\sigma^{2}}{2}\right) d t+\sigma d B(t)\right) .
$$

Due to the complexity of $\beta_{1}^{1}, \beta_{2}^{1}, \beta_{1}^{2}$ and $\beta_{2}^{2}$ shown in (11) - (14), SDEs of the wealth processes

$$
d w_{t}^{1}=\beta_{1}^{1}\left(w_{t}, \lambda_{k}\right) d t+\beta_{2}^{1}\left(w_{t}, \lambda_{k}\right) d B(t)
$$

and

$$
d w_{t}^{2}=\beta_{1}^{2}\left(w_{t}, \lambda_{k}\right) d t+\beta_{2}^{2}\left(w_{t}, \lambda_{k}\right) d B(t)
$$

cannot be solved analytically. We used the MATLAB ${ }^{\circledR}$ software to simulate the Brownian Motion and find the numerical solutions of these SDEs ${ }^{1}$. In the simulation, the parameters' values in the dividend processes are set as follow. Interest rate $(r)$ is set to be $7 \%$ while $\alpha$ and $\sigma$ are set to be 0.07 and 0.2 respectively. The consumption rate $\left(\lambda_{0}\right)$ is equal to 0.1 to be used in the simulation. Moreover, we consider the simulation in 3 scenarios depending on the initial wealth of the two investors $w_{0}^{1}$ and $w_{0}^{2}$. In the first case, we simulate with the initial wealth of the two investors assumed to be equal, that is $w_{0}^{1}=w_{0}^{2}=1$. In the second case, the initial wealth of the second investor is more than the first one, say $w_{0}^{1}=1$ and $w_{0}^{2}=2$. Finally, the initial wealth of the first investor is more than the second one; we use $w_{0}^{1}=1$ and $w_{0}^{2}=0.6$.

In each case, we evaluate the market share of each investors by

$$
r_{t}^{i}=\frac{w_{t}^{i}}{\sum_{i=1}^{I} w_{t}^{i}}
$$

with the different portfolio rules of both investors. By fixing $\lambda_{1}^{1}$; the proportion of wealth investor 1 invests in the bond, we plot the market shares of the

\footnotetext{
${ }^{1}$ The MATLAB code is not provided in this paper. However, it is available on request.
} 
investors corresponding to $\lambda_{1}^{2}$; the proportion of wealth investor 2 invests in bond, which is increasing by 0.1 from 0 to $1-\lambda_{0}$. Note that there is not a case when $\lambda_{1}^{1}=\lambda_{1}^{2}=0$ and $\lambda_{1}^{1}=\lambda_{1}^{2}=1-\lambda_{0}$, because both of them lead to $p_{t}^{k}=0$.

For the first scenario, $w_{0}^{1}=w_{0}^{2}=1$; that is $\left(r_{0}^{1}, r_{0}^{2}\right)=(0.5,0.5)$, the following results are found. If the investor invests his total money in stock; $\lambda_{1}^{i}=0$, another investor dominates the market for all his strategies. (Recall that there is not a case that both investors invest all their money either in bond or stock.) In addition, if both agents use the same strategies, their market shares will not be different. That is both $r_{T}^{1}$ and $r_{T}^{2}$ are closed to 0.5. We can interpret that the agent will dominate the market if he invests in bond more than another agent. However, for all strategies of both investors we have tried, there is not a case where any investor can obtain total market share. The maximum of market share one can reach is around 0.7. It means that if both investors start with the same amount of money, it does not matter what strategies they use, they both survive in the market. Some samples of the simulation are illustrated in Figure 1, where the dashed line (- - ) denotes $r_{t}^{1}$ and the line with asterisk $\left(-^{*}\right)$ represents $r_{t}^{2}$.

The second scenario is $w_{0}^{1}=1$ and $w_{0}^{2}=2$; that is $\left(r_{0}^{1}, r_{0}^{2}\right)=(0.3333,0.6667)$. Some samples of the simulation are demonstrated in Figure 2. We found that the second investor dominates the market for all cases of the first investor's strategies, except the case when $\lambda_{1}^{1}=0.9$ and $\lambda_{1}^{2}=0$ as shown in Figure 2(c). It is the case that the first agent invests all his money in the bond while the second agent invests all his money in stock. If this case happens, the market shares of both investors are quite similar (in fact $r_{T}^{1}$ and $r_{T}^{2}$ are closed to 0.5$)$. The best strategy of the second trader is to invest all his budget in bonds $\left(\lambda_{1}^{2}=0.9\right)$, because this strategy leads him to gain more market share than another no matter what the first investor's strategies are. For all $\lambda_{1}^{1}$ and $0 \leq \lambda_{1}^{2} \leq 0.8$, the market shares are changed in small intervals.

The third scenario is $w_{0}^{1}=1$ and $w_{0}^{2}=0.6$; that is $\left(r_{0}^{1}, r_{0}^{2}\right)=(0.625,0.375)$. Samples of the simulation are presented in Figure 3. The simulation illustrates that the first agent dominates the market for all strategies of the second agent, except two cases which are $\left(\lambda_{1}^{1}, \lambda_{1}^{2}\right)=(0,0.9)$ and $(0.1,0.9)$. In the first case; Figure 3(a), the agent 2 dominates the market while another case; Figure 3(b), both agents' market shares are similar $\left(r_{T}^{1}\right.$ and $r_{T}^{2}$ are closed to 0.5). This scenario seems to be in opposition to the second scenario, the difference is, there are some portfolio rules which lead agent 1 to obtain a whole market share and agent 2 going bankrupt in the market; i.e. $\left(r_{T}^{1}, r_{T}^{2}\right)$ is close to $(1,0)$. For the second agent; the higher $\lambda_{1}^{2}$, the higher $r_{T}^{2}$. In most cases, $r_{T}^{2}$ is maximal when $\lambda_{1}^{2}=0.9$ except only one case when $\lambda_{1}^{1}$ is also equal to 0.9 . If $\lambda_{1}^{1}=0.9$, maximum $r_{T}^{2}$ comes from $\lambda_{1}^{2}=0$. 


\section{Appendix}

\subsection{Technical Computations}

This section contains the details of the computations as shown in (11) - (14). From the form of wealth processes we set up and Itô formula, we can see that $\left(d w_{t}^{i}\right)^{2}=\left(\beta_{2}^{i}\right)^{2} d t$. After we substitute for $d D_{t}^{k}, d p_{t}^{k}$ and $d w_{t}^{i}$, and $d \theta_{t, k}^{i}$ from (9) using the previous relation for $\left(d w_{t}^{i}\right)^{2}$ in the wealth processes (5) and rearrange the equations, the following four equations are obtained for solving for $\beta_{1}^{1}, \beta_{2}^{1}, \beta_{1}^{2}$ and $\beta_{2}^{2}$.

$$
\begin{aligned}
& \beta_{1}^{1}=r\left(\frac{\lambda_{1}^{1} w_{t}^{1}}{\lambda_{1}^{1} w_{t}^{1}+\lambda_{1}^{2} w_{t}^{2}}\right)+\left(\lambda_{1}^{1} \beta_{1}^{1}+\lambda_{1}^{2} \beta_{1}^{2}\right)\left(\frac{\lambda_{1}^{1} w_{t}^{1}}{\lambda_{1}^{1} w_{t}^{1}+\lambda_{1}^{2} w_{t}^{2}}\right) \\
& +\left\{\left(D_{t}^{1}+\lambda_{1}^{1} w_{t}^{1}+\lambda_{1}^{2} w_{t}^{2}\right)\left(\frac{\left(\lambda_{1}^{1} w_{t}^{1}+\lambda_{1}^{2} w_{t}^{2}\right)\left(\lambda_{1}^{1}\right)-\left(\lambda_{1}^{1} w_{t}^{1}\right)\left(\lambda_{1}^{1}+\lambda_{1}^{2}\right)}{\left(\lambda_{1}^{1} w_{t}^{1}+\lambda_{1}^{2} w_{t}^{2}\right)^{2}}\right)\right. \\
& \left.\left(\beta_{1}^{1}-\frac{\left(\beta_{2}^{1}\right)^{2}\left(\lambda_{1}^{1}+\lambda_{1}^{2}\right)}{\lambda_{1}^{1} w_{t}^{1}+\lambda_{1}^{2} w_{t}^{2}}\right)\right\} \\
& +\alpha\left(\frac{\lambda_{2}^{1} w_{t}^{1}}{\lambda_{2}^{1} w_{t}^{1}+\lambda_{2}^{2} w_{t}^{2}}\right)+\left(\lambda_{2}^{1} \beta_{1}^{1}+\lambda_{2}^{2} \beta_{1}^{2}\right)\left(\frac{\lambda_{2}^{1} w_{t}^{1}}{\lambda_{2}^{1} w_{t}^{1}+\lambda_{2}^{2} w_{t}^{2}}\right) \\
& +\left\{\left(D_{t}^{2}+\lambda_{2}^{1} w_{t}^{1}+\lambda_{2}^{2} w_{t}^{2}\right)\left(\frac{\left(\lambda_{2}^{1} w_{t}^{1}+\lambda_{2}^{2} w_{t}^{2}\right)\left(\lambda_{2}^{1}\right)-\left(\lambda_{2}^{1} w_{t}^{1}\right)\left(\lambda_{2}^{1}+\lambda_{2}^{2}\right)}{\left(\lambda_{2}^{1} w_{t}^{1}+\lambda_{2}^{2} w_{t}^{2}\right)^{2}}\right)\right. \\
& \left.\left(\beta_{1}^{1}-\frac{\left(\beta_{2}^{1}\right)^{2}\left(\lambda_{2}^{1}+\lambda_{2}^{2}\right)}{\lambda_{2}^{1} w_{t}^{1}+\lambda_{2}^{2} w_{t}^{2}}\right)\right\} \\
& \beta_{1}^{2}=r\left(\frac{\lambda_{1}^{2} w_{t}^{2}}{\lambda_{1}^{1} w_{t}^{1}+\lambda_{1}^{2} w_{t}^{2}}\right)+\left(\lambda_{1}^{1} \beta_{1}^{1}+\lambda_{1}^{2} \beta_{1}^{2}\right)\left(\frac{\lambda_{1}^{2} w_{t}^{2}}{\lambda_{1}^{1} w_{t}^{1}+\lambda_{1}^{2} w_{t}^{2}}\right) \\
& +\left\{\left(D_{t}^{1}+\lambda_{1}^{1} w_{t}^{1}+\lambda_{1}^{2} w_{t}^{2}\right)\left(\frac{\left(\lambda_{1}^{1} w_{t}^{1}+\lambda_{1}^{2} w_{t}^{2}\right)\left(\lambda_{1}^{2}\right)-\left(\lambda_{1}^{2} w_{t}^{2}\right)\left(\lambda_{1}^{1}+\lambda_{1}^{2}\right)}{\left(\lambda_{1}^{1} w_{t}^{1}+\lambda_{1}^{2} w_{t}^{2}\right)^{2}}\right)\right. \\
& \left.\left(\beta_{1}^{2}-\frac{\left(\beta_{2}^{2}\right)^{2}\left(\lambda_{1}^{1}+\lambda_{1}^{2}\right)}{\lambda_{1}^{1} w_{t}^{1}+\lambda_{1}^{2} w_{t}^{2}}\right)\right\} \\
& +\alpha\left(\frac{\lambda_{2}^{2} w_{t}^{2}}{\lambda_{2}^{1} w_{t}^{1}+\lambda_{2}^{2} w_{t}^{2}}\right)+\left(\lambda_{2}^{1} \beta_{1}^{1}+\lambda_{2}^{2} \beta_{1}^{2}\right)\left(\frac{\lambda_{2}^{2} w_{t}^{2}}{\lambda_{2}^{1} w_{t}^{1}+\lambda_{2}^{2} w_{t}^{2}}\right) \\
& +\left\{\left(D_{t}^{2}+\lambda_{2}^{1} w_{t}^{1}+\lambda_{2}^{2} w_{t}^{2}\right)\left(\frac{\left(\lambda_{2}^{1} w_{t}^{1}+\lambda_{2}^{2} w_{t}^{2}\right)\left(\lambda_{2}^{2}\right)-\left(\lambda_{2}^{2} w_{t}^{2}\right)\left(\lambda_{2}^{1}+\lambda_{2}^{2}\right)}{\left(\lambda_{2}^{1} w_{t}^{1}+\lambda_{2}^{2} w_{t}^{2}\right)^{2}}\right)\right. \\
& \left.\left(\beta_{1}^{2}-\frac{\left(\beta_{2}^{2}\right)^{2}\left(\lambda_{2}^{1}+\lambda_{2}^{2}\right)}{\lambda_{2}^{1} w_{t}^{1}+\lambda_{2}^{2} w_{t}^{2}}\right)\right\}
\end{aligned}
$$




$$
\begin{aligned}
\beta_{2}^{1}= & \left(\lambda_{1}^{1} \beta_{2}^{1}+\lambda_{1}^{2} \beta_{2}^{2}\right)\left(\frac{\lambda_{1}^{1} w_{t}^{1}}{\lambda_{1}^{1} w_{t}^{1}+\lambda_{1}^{2} w_{t}^{2}}\right) \\
& +\left(D_{t}^{1}+\lambda_{1}^{1} w_{t}^{1}+\lambda_{1}^{2} w_{t}^{2}\right)\left[\frac{\left(\lambda_{1}^{1} w_{t}^{1}+\lambda_{1}^{2} w_{t}^{2}\right)\left(\lambda_{1}^{1}\right)-\left(\lambda_{1}^{1} w_{t}^{1}\right)\left(\lambda_{1}^{1}+\lambda_{1}^{2}\right)}{\left(\lambda_{1}^{1} w_{t}^{1}+\lambda_{1}^{2} w_{t}^{2}\right)^{2}}\right] \beta_{2}^{1} \\
& +\sigma\left(\frac{\lambda_{2}^{1} w_{t}^{1}}{\lambda_{2}^{1} w_{t}^{1}+\lambda_{2}^{2} w_{t}^{2}}\right)+\left(\lambda_{2}^{1} \beta_{2}^{1}+\lambda_{2}^{2} \beta_{2}^{2}\right)\left(\frac{\lambda_{2}^{1} w_{t}^{1}}{\lambda_{2}^{1} w_{t}^{1}+\lambda_{2}^{2} w_{t}^{2}}\right) \\
& +\left(D_{t}^{2}+\lambda_{2}^{1} w_{t}^{1}+\lambda_{2}^{2} w_{t}^{2}\right)\left[\frac{\left(\lambda_{2}^{1} w_{t}^{1}+\lambda_{2}^{2} w_{t}^{2}\right)\left(\lambda_{2}^{1}\right)-\left(\lambda_{2}^{1} w_{t}^{1}\right)\left(\lambda_{2}^{1}+\lambda_{2}^{2}\right)}{\left(\lambda_{2}^{1} w_{t}^{1}+\lambda_{2}^{2} w_{t}^{2}\right)^{2}}\right] \beta_{2}^{1} \\
\beta_{2}^{2}= & \left(\lambda_{1}^{1} \beta_{2}^{1}+\lambda_{1}^{2} \beta_{2}^{2}\right)\left(\frac{\lambda_{1}^{2} w_{t}^{2}}{\lambda_{1}^{1} w_{t}^{1}+\lambda_{1}^{2} w_{t}^{2}}\right) \\
& +\left(D_{t}^{1}+\lambda_{1}^{1} w_{t}^{1}+\lambda_{1}^{2} w_{t}^{2}\right)\left[\frac{\left(\lambda_{1}^{1} w_{t}^{1}+\lambda_{1}^{2} w_{t}^{2}\right)\left(\lambda_{1}^{2}\right)-\left(\lambda_{1}^{2} w_{t}^{2}\right)\left(\lambda_{1}^{1}+\lambda_{1}^{2}\right)}{\left(\lambda_{1}^{1} w_{t}^{1}+\lambda_{1}^{2} w_{t}^{2}\right)^{2}}\right] \beta_{2}^{2} \\
& +\sigma\left(\frac{\lambda_{2}^{2} w_{t}^{2}}{\lambda_{2}^{1} w_{t}^{1}+\lambda_{2}^{2} w_{t}^{2}}\right)+\left(\lambda_{2}^{1} \beta_{2}^{1}+\lambda_{2}^{2} \beta_{2}^{2}\right)\left(\frac{\lambda_{2}^{2} w_{t}^{2}}{\lambda_{2}^{1} w_{t}^{1}+\lambda_{2}^{2} w_{t}^{2}}\right) \\
& +\left(D_{t}^{2}+\lambda_{2}^{1} w_{t}^{1}+\lambda_{2}^{2} w_{t}^{2}\right)\left[\frac{\left(\lambda_{2}^{1} w_{t}^{1}+\lambda_{2}^{2} w_{t}^{2}\right)\left(\lambda_{2}^{2}\right)-\left(\lambda_{2}^{2} w_{t}^{2}\right)\left(\lambda_{2}^{1}+\lambda_{2}^{2}\right)}{\left(\lambda_{2}^{1} w_{t}^{1}+\lambda_{2}^{2} w_{t}^{2}\right)^{2}}\right] \beta_{2}^{2}
\end{aligned}
$$

First, we solve for $\beta_{2}^{1}$ and $\beta_{2}^{2}$ from (17) and (18)

$$
\begin{aligned}
\beta_{2}^{1}+\beta_{2}^{2}= & \left(\lambda_{1}^{1} \beta_{2}^{1}+\lambda_{1}^{2} \beta_{2}^{2}\right)\left(\frac{\lambda_{1}^{1} w_{t}^{1}+\lambda_{1}^{2} w_{t}^{2}}{\lambda_{1}^{1} w_{t}^{1}+\lambda_{1}^{2} w_{t}^{2}}\right) \\
& +\frac{\left(D_{t}^{1}+\lambda_{1}^{1} w_{t}^{1}+\lambda_{1}^{2} w_{t}^{2}\right)\left(\lambda_{1}^{1} \lambda_{1}^{2}\right)\left(w_{t}^{1}-w_{t}^{2}\right)\left(\beta_{2}^{2}-\beta_{2}^{1}\right)}{\left(\lambda_{1}^{1} w_{t}^{1}+\lambda_{1}^{2} w_{t}^{2}\right)^{2}} \\
& +\sigma\left(\frac{\lambda_{2}^{1} w_{t}^{1}+\lambda_{2}^{2} w_{t}^{2}}{\lambda_{2}^{1} w_{t}^{1}+\lambda_{2}^{2} w_{t}^{2}}\right)+\left(\lambda_{2}^{1} \beta_{2}^{1}+\lambda_{2}^{2} \beta_{2}^{2}\right)\left(\frac{\lambda_{2}^{1} w_{t}^{1}+\lambda_{2}^{2} w_{t}^{2}}{\lambda_{2}^{1} w_{t}^{1}+\lambda_{2}^{2} w_{t}^{2}}\right) \\
& +\frac{\left(D_{t}^{2}+\lambda_{2}^{1} w_{t}^{1}+\lambda_{2}^{2} w_{t}^{2}\right)\left(\lambda_{2}^{1} \lambda_{2}^{2}\right)\left(w_{t}^{1}-w_{t}^{2}\right)\left(\beta_{2}^{2}-\beta_{2}^{1}\right)}{\left(\lambda_{2}^{1} w_{t}^{1}+\lambda_{2}^{2} w_{t}^{2}\right)^{2}}
\end{aligned}
$$

By using $P_{k}=\lambda_{k}^{1} w_{t}^{1}+\lambda_{k}^{2} w_{t}^{2}, W=w_{t}^{1}-w_{t}^{2}, A^{k}=\lambda_{k}^{1} \lambda_{k}^{2} \frac{D_{t}^{k}+P_{k}}{\left(P_{k}\right)^{2}}$ and $A=A^{1}+A^{2}$, we get

$$
\beta_{2}^{1}+\beta_{2}^{2}=\left[\left(\lambda_{1}^{1}+\lambda_{2}^{1}\right)-W A\right] \beta_{2}^{1}+\left[\left(\lambda_{1}^{2}+\lambda_{2}^{2}\right)+W A\right] \beta_{2}^{2}+\sigma
$$




$$
\beta_{2}^{2}=\frac{W A+\lambda_{0}}{W A-\lambda_{0}} \beta_{2}^{1}-\frac{\sigma}{W A-\lambda_{0}}
$$

Inserting (19) in (17) yields

$$
\begin{aligned}
\beta_{2}^{1}= & \left(\frac{\lambda_{1}^{1} \lambda_{1}^{2} w_{t}^{1}}{P_{1}}+\frac{\lambda_{2}^{1} \lambda_{2}^{2} w_{t}^{1}}{P_{2}}\right)\left[\frac{W A+\lambda_{0}}{W A-\lambda_{0}} \beta_{2}^{1}-\frac{\sigma}{W A-\lambda_{0}}\right] \\
& +\frac{\left(\lambda_{1}^{1}\right)^{2} w_{t}^{1}}{P_{1}} \beta_{2}^{1}+\frac{\left(\lambda_{2}^{1}\right)^{2} w_{t}^{1}}{P_{2}} \beta_{2}^{1}-\frac{D_{t}^{1}+P_{1}}{\left(P_{1}\right)^{2}}\left(\lambda_{1}^{1} \lambda_{1}^{2}\right)\left(w_{t}^{1}-w_{t}^{2}\right) \beta_{2}^{1} \\
& -\frac{D_{t}^{2}+P_{2}}{\left(P_{2}\right)^{2}}\left(\lambda_{2}^{1} \lambda_{2}^{2}\right)\left(w_{t}^{1}-w_{t}^{2}\right) \beta_{2}^{1}+\sigma \frac{\lambda_{2}^{1} w_{t}^{1}}{P_{2}}
\end{aligned}
$$

That is

$$
\beta_{2}^{1}=\frac{\sigma w_{t}^{1}\left(\frac{\lambda_{2}^{1}}{P_{2}}-\frac{C}{W A-\lambda_{0}}\right)}{1-w_{t}^{1} C\left(\frac{W A+\lambda_{0}}{W A-\lambda_{0}}\right)-w_{t}^{1}\left(\frac{\left(\lambda_{1}^{1}\right)^{2}}{P_{1}}+\frac{\left(\lambda_{2}^{1}\right)^{2}}{P_{2}}\right)+W A}
$$

where $C=\frac{\lambda_{1}^{1} \lambda_{1}^{2}}{P_{1}}+\frac{\lambda_{2}^{1} \lambda_{2}^{2}}{P_{2}}$.

Next, using (15) and (16) solve for $\beta_{1}^{1}$ and $\beta_{1}^{2}$.

$$
\begin{aligned}
\beta_{1}^{1}+\beta_{1}^{2}= & r\left(\frac{\lambda_{1}^{1} w_{t}^{1}+\lambda_{1}^{2} w_{t}^{2}}{P_{1}}\right)+\alpha\left(\frac{\lambda_{2}^{1} w_{t}^{1}+\lambda_{2}^{2} w_{t}^{2}}{P_{2}}\right) \\
& +\left(\lambda_{1}^{1} \beta_{1}^{1}+\lambda_{1}^{2} \beta_{1}^{2}\right)\left(\frac{\lambda_{1}^{1} w_{t}^{1}+\lambda_{1}^{2} w_{t}^{2}}{P_{1}}\right)+\left(\lambda_{2}^{1} \beta_{1}^{1}+\lambda_{2}^{2} \beta_{1}^{2}\right)\left(\frac{\lambda_{2}^{1} w_{t}^{1}+\lambda_{2}^{2} w_{t}^{2}}{P_{2}}\right) \\
& +\frac{\left(D_{t}^{1}+P_{1}\right)\left(\lambda_{1}^{1} \lambda_{1}^{2}\right)\left(w_{t}^{1}-w_{t}^{2}\right)}{\left(P_{1}\right)^{2}}\left[\left(\beta_{1}^{2}-\beta_{1}^{1}\right)-\left\{\left(\beta_{2}^{2}\right)^{2}-\left(\beta_{2}^{1}\right)^{2}\right\}\left(\frac{\lambda_{1}^{1}+\lambda_{1}^{2}}{P_{1}}\right)\right] \\
& +\frac{\left(D_{t}^{2}+P_{2}\right)\left(\lambda_{2}^{1} \lambda_{2}^{2}\right)\left(w_{t}^{1}-w_{t}^{2}\right)}{\left(P_{2}\right)^{2}}\left[\left(\beta_{1}^{2}-\beta_{1}^{1}\right)-\left\{\left(\beta_{2}^{2}\right)^{2}-\left(\beta_{2}^{1}\right)^{2}\right\}\left(\frac{\lambda_{2}^{1}+\lambda_{2}^{2}}{P_{2}}\right)\right]
\end{aligned}
$$

$$
\begin{aligned}
\beta_{1}^{1}+\beta_{1}^{2}= & r+\alpha+\left(\lambda_{1}^{1}+\lambda_{2}^{1}\right) \beta_{1}^{1}+\left(\lambda_{1}^{2}+\lambda_{2}^{2}\right) \beta_{1}^{2}+W\left(A^{1}+A^{2}\right)\left(\beta_{1}^{2}-\beta_{1}^{1}\right) \\
& -W\left[\frac{A^{1}\left(\lambda_{1}^{1}+\lambda_{1}^{2}\right)}{P_{1}}+\frac{A^{2}\left(\lambda_{2}^{1}+\lambda_{2}^{2}\right)}{P_{2}}\right]\left[\left(\beta_{2}^{2}\right)^{2}-\left(\beta_{2}^{1}\right)^{2}\right]
\end{aligned}
$$




$$
\begin{aligned}
\beta_{1}^{2}= & \left(\frac{W A+\lambda_{0}}{W A-\lambda_{0}}\right) \beta_{1}^{1}+\frac{W\left(A^{1} E^{1}+A^{2} E^{2}\right)}{W A-\lambda_{0}}\left[\left(\beta_{2}^{2}\right)^{2}-\left(\beta_{2}^{1}\right)^{2}\right] \\
& -\frac{r}{W A-\lambda_{0}}-\frac{\alpha}{W A-\lambda_{0}}
\end{aligned}
$$

where $E^{k}=\frac{\lambda_{k}^{1}+\lambda_{k}^{2}}{P_{k}}$.

Finally,

$$
\begin{aligned}
\beta_{1}^{1}= & \frac{1}{1-w_{t}^{1} C\left(\frac{W A+\lambda_{0}}{W A-\lambda_{0}}\right)-w_{t}^{1}\left(\frac{\left(\lambda_{1}^{1}\right)^{2}}{P_{1}}+\frac{\left(\lambda_{2}^{1}\right)^{2}}{P_{2}}\right)+W A} \\
& {\left[\frac{w_{t}^{1} C}{W A-\lambda_{0}}\left\{W\left(A^{1} E^{1}+A^{2} E^{2}\right)\left[\left(\beta_{2}^{2}\right)^{2}-\left(\beta_{2}^{1}\right)^{2}\right]-r-\alpha\right\}\right.} \\
& \left.+r\left(\frac{\lambda_{1}^{1} w_{t}^{1}}{P_{1}}\right)+\alpha\left(\frac{\lambda_{2}^{1} w_{t}^{1}}{P_{2}}\right)+W\left(A^{1} E^{1}+A^{2} E^{2}\right)\left(\beta_{2}^{1}\right)^{2}\right]
\end{aligned}
$$




\section{$5.2 \quad$ Figures}
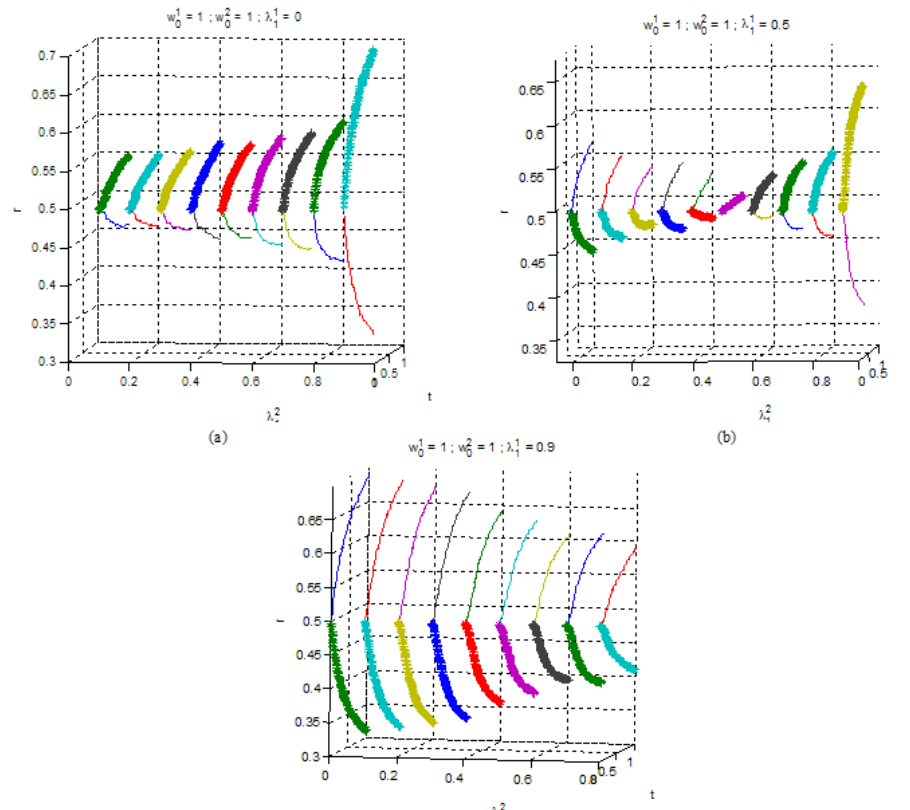

(c)

Figure 1: 


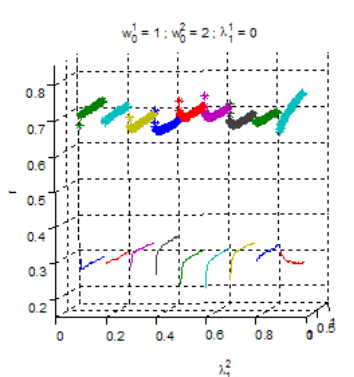

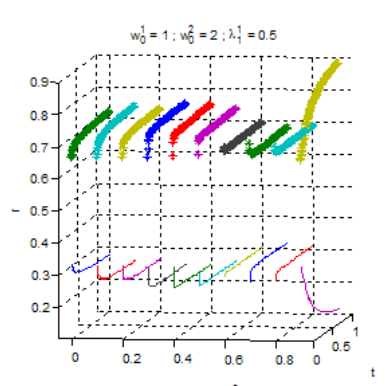

(b)

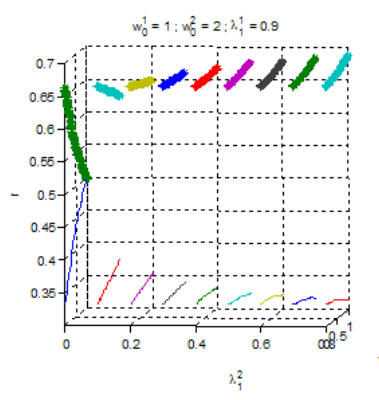

(c)

Figure 2: 


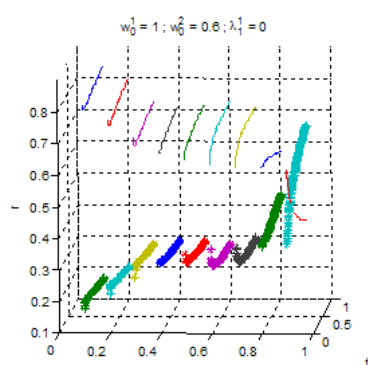

(2.

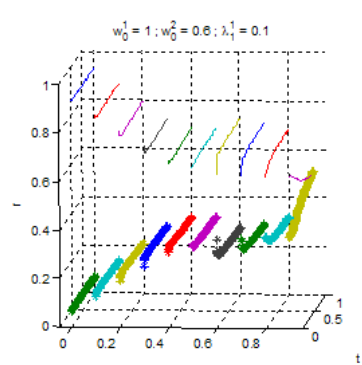

(b)

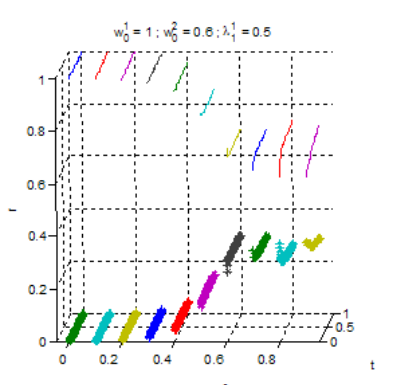

(c)

Figure 3:

ACKNOWLEDGEMENTS. The first author would like to acknowledge the Royal Thai Government for their financial support.

\section{References}

[1] C.O. Mahony, The Numerical Analysis of Stochastic Differential Equations, 2006.

[2] D.J.Higham, An Algorithmic Introduction to Numerical Simulation of Stochastic Differential Equations, SIAM Review 43(3) (2001), 525-546.

[3] F. Chang, Stochastic Optimization in Continuous Time, Cambridge University Press, 2004.

[4] H. Gilsing and T. Shardlow, SDELab: Stochastic Differential Equations with MATLAB, 2005.

[5] I.V. Evstigneev, T. Hens, and K. R. Schenk-Hoppé, Evolutionary Stable Stock Market, Economic Theory 27(2) (2006), 449-468.

[6] J.S. Dagpunar, Simulation and Monte Carlo with Applications in Finance and MCMC, John Wiley \& Sons, Ltd., 2007. 
[7] R. Merton, Continuous-time Finance, Blackwell Publishers, 1992.

[8] S.E. Shreve, Stochastic Calculus for Finance: Continuous-time Models: v.2, Springer-Verlag New York Inc., 2004.

[9] S.N. Neftci, An introduction to the Mathematics of Financial Derivatives, 2nd Edition, Academic Press, 2000.

[10] T. Bjork, Arbitrage Theory in Continuous Time, Oxford University Press, 2nd Edition, 2004.

[11] Z. Yang and C.O. Ewald, Continuous Time Evolutionary Market Dynamics: The Case of Fix-Mix Strategies, Investment Management and Financial Innovation 5(1) (2008).

Received: February 29, 2008 